\title{
The millennial challenge: Modelling the agentic self in context
}

\author{
Todd D. Little \\ Yale University, New Haven, USA
}

\begin{abstract}
Behind every challenge is an opportunity. As a milestone for both societal and scientific development, the new millennium highlights the growing challenge of change and diversity. Modelling the agentic self in context is the opportunity it reveals. Developmentalists can seize this opportunity by increasing our emphasis on three aspects of developmental research. First, developmental research can examine the influence of context on development more aggressively to test the generality of developmental mechanisms. Second, developmental research can integrate an organismic perspective on human development more explicitly to account for the role of human agency in development. Third, developmental research can apply advanced statistical and analysis methods more sophisticatedly to effectively model the agentic self as it evolves across time and context.
\end{abstract}

The term "new millennium" often connotes fervent images of change and uncertainty. Even casual observation reinforces the notion that many (if not most) cultural, societal, social, and familial structures have dramatically transformed during the latter part of this century. Not surprisingly, the trends indicate that we can expect continued diversification as we enter the next century (e.g., post-retirement lifestyles, familial constellations, educational choices, social norms, career directions, and so on will likely continue to become more varied and differentiated). Consequent to these changes, every phase of the lifespan will, of necessity, be faced with new and diverse challenges to successful development. At the same time, however, many of these defining features of society todayand tomorrow, as we traverse the millennial milestone-also reveal valuable opportunities for developmental psychology.

As I see it, developmental psychology is in a unique position to capitalise on the opportunities that the changing sociocultural structures offer and, thereby make significant advances, by focusing on three related themes. First, developmental psychology should examine the influence of context on development more aggressively (e.g., Bronfenbrenner, 1995; Lerner, 1996). The remarkable resurgence of crosscultural designs, for example, already indicates that we are moving in this direction. To truly capitalise on the benefits of a contextual perspective, however, even better articulated theories of and compelling designs for contextual studies will need to be implemented. Second, developmental psychology should seek to better understand the interrelations among the varied features of the self system and the roles of various selfregulatory processes in development (e.g., motives, goals, volitional control mechanisms, mental representations, subjective interpretations, and so on). An organismic perspective on the individual offers a useful starting point from which to fully unpack the relationships among the qualities and quantities of the agentic self (e.g., Little, Hawley, Henrich, \& Marsland, in press; Magnusson \& Cairns, 1996; Ryan, 1993;
Ryan, Sheldon, Kasser, \& Deci, 1996). Third, developmental psychology should seek to further increase the methodological sophistication by which the interplay of context and self is disentangled. The latter quarter of this century saw the introduction of an extremely powerful and versatile family of statistical modelling tools (e.g., general structural equation modelling techniques, including specific instantiations such as multilevel, growth-curve, and multiple-group models; Little, Schnabel, \& Baumert, 2000; Marcoulides \& Schumacker, 1996). These modelling tools are continuing to be refined and will soon offer even greater flexibility for researchers to ask and answer the types of complex questions that are needed for understanding developmental processes across contexts.

\section{The role of context}

A contextual perspective explicitly acknowledges that every developmental epoch represents a unique constellation of influences (Bronfenbrenner, 1995; Lerner, 1995, 1996). Contexts provide both affordances and constraints on the range of individual actions and behaviours that can be expressed. Although some mechanisms of development are clearly universal (i.e., transcend context), many of the mechanisms that will ultimately account for the diversity of human behaviour across developmental contexts and the life course will likely be context-specific mechanisms or, at a minimum, mechanisms that are highly moderated by context (i.e., mechanisms that function only in interaction with extrapersonal features). Taken together, both the pre- and post-millennial changes in the various social structures amount to unprecedented variability among the contexts in which development unfolds. As a result, a primary need of developmental psychology will be to examine the processes of change, growth, and transformation as they unfold within and between these varied contexts.
Correspondence should be addressed to Todd Little, Department of Psychology, Yale University, PO Box 208205, New Haven, CT 06520-8205, USA; e-mail: Todd.Little@Yale.edu.

I would like to express my deepest gratitude to the many who have influenced my thinking about developmental psychology, in particular, Paul Baltes, John
Nesselroade, Richard Ryan, Keith Widaman, and my former colleagues at the Max Planck Institute for Human Development in Berlin. I am also grateful for the feedback and comments of my new colleagues in the Department of Psychology at Yale University. 
Generally speaking, a given context reflects a specific constellation of features (i.e., where intra-, inter-, and extrapersonal features converge). If carefully contrasted, these multilayered features can provide a quasi-experimental manipulation of many potential mechanisms that can shape and mould individual development. Given that the features in which developmental processes occur can be systematically varied, contextual designs can uncover the working mechanisms that influence development and they can dramatically increase our universe of generalisation. For instance, comparing individual development across varying contexts can highlight which facets of the self-system are independent of the variations and which are not (e.g., Little, 1998). Similarly, comparing the same individual across numerous contexts would reveal which processes of the agentic self function similarly (transcend context) and which function uniquely as a result of context-specific constraints or affordances (Mischel \& Shoda, 1995). In other words, well-conceived large-scale contextual studies can systematically vary and thereby quasiexperimentally isolate specific features of both contexts and individuals in order to derive the nature and generality of their respective influences on developmental outcomes.

As I see it, instead of simply documenting group-level differences in development across various contexts, a fruitful goal of contextual approaches would be to attempt to reduce context to a set of individual-differences variables that can effectively explain away the group-level differences of the individuals in a given context. In other words, by explicitly measuring the defining features of various contexts at the level of the individual, greater precision in isolating the working mechanisms of change, growth, and transformation would be gained. For example, instead of characterising two sociocultural contexts as individualistic versus collectivistic, a researcher could measure the degree to which each individual in each context endorses individualistic and collectivistic values and ideals (i.e., one's personal balance of the cultural values). After controlling for these individual-differences variables, one could then examine whether group-level differences still remain.

The contextual perspective also encourages a typological approach to understanding individual development. Given that all developmental processes likely do not function in the same way for all individuals, one could reasonably expect differing constellations of cognitions and behaviours that characterise subsets of individuals. These subgroups or typologies would also be characterised by a range of individual differences within them. In this sense, a typological profile would be considered a specific developmental context, with unique affordances and constraints on behaviour. For example, the profile of an individual who is both high on individualistic values and collectivistic values suggests constraints on behaviour that would not be the same for an individual who is low on both dimensions or high on one but medium or low on the other. However, especially when typologies are more theory-driven rather than data-driven, a typological approach can be quite useful for understanding developmental processes (see e.g., Hawley, Pasupathi, \& Little, 1999; Smith \& Baltes, 1999).

\section{The role of an organismic perspective}

An organismic approach to understanding the developing individual involves an explicit focus on the interface between the self and context (Hawley, 1999; Little et al., in press; Ryan et al., 1996). The individual is viewed as an integrated organism that both influences and is influenced by the contexts in which he/she acts and develops. Given that many research accounts seem to rely on implicit assumptions about the nature of the individual, more rapid and efficient progress will come about if we make explicit the larger models of development that drive various microtheories (Shanahan, Valsiner, \& Gottlieb, 1997). For example, to what degree does a theory's model assume that humans are active agents in and contributors to their own development or merely reactive pawns in a larger environmentally driven macrocosm? At the heart of an organismic perspective is the presumption that individuals are active agents and contributors to their own development, but that the agentic self must negotiate the affordances and constraints of each context.

An organismic perspective also entails a systems approach wherein the functioning of one system influences the functioning of another (Gottlieb, 1997). With the subsystems yoked (seemingly inextricably), research programmes will be optimally informative when various subsystems are examined together rather than in isolation (Magnusson \& Cairns, 1996). Such a view requires greater investment in planning a data collection protocol and greater investment in procuring appropriate samples of participants (e.g., samples derived on the basis of theoretical considerations rather than convenience).

Not only does an organismic perspective encourage broadbased studies of the agentic self, but also it supports a concerted focus on successful developmental outcomes. In particular, as each child begins to discover who he/she is and what he/she is capable of, the evolving competence system gives rise to a developing sense of personal agency-the agentic self. The resulting systems of action-control beliefs and behaviours provide a developmental foundation that is called upon to negotiate various developmental tasks and challenges throughout the life course. In facing these challenges, an agentic individual is primarily the origin of his/her actions, has high aspirations, perseveres in the face of obstacles, sees more and varied options, learns from failures, has a greater sense of well-being, and so on. On the other hand, a nonagentic individual is primarily the pawn of unknown extrapersonal influences, has low aspirations, is hindered with problemsolving blinders, and often feels helpless and unempowered (Little, 1998, Little et al., in press; Ryan et al., 1996; Skinner, 1995; Weisz, 1990).

Although the range of individual differences between these extreme profiles is wide, it seems warranted to focus our attention on those factors that optimise the agentic self, including feelings of autonomy, competence, and relatedness (Ryan et al., 1996). By explicitly examining the role of agency in development, we can identify those features of both individuals and contexts that maximally contribute to the agentic self and successful development along the life course (Lewis, 1997). Understanding the possible avenues of development that lead to the emergence of agentic and efficacious individuals can reap great rewards in terms of the productivity and well-being of the millennial generation, especially given its unique relationship to this historic milestone.

\section{The modelling theme}

The contextual and organismic perspectives necessitate sophisticated statistical and methodological tools be used to 
disentangle (model) the various sources of influence. A primary goal of a modelling perspective is to make strong tests of underlying theoretical models (i.e., bring implicit assumptions into the explicit realm of model specification). A modelling perspective is not limited to structural modelling techniques - it is equally at home within the framework of more classical, regression-based techniques (see e.g., Widaman, 2000). From a modelling perspective, statistical procedures are not mechanistic ends in themselves (i.e., fixed and rule-bound), but rather are flexible tools that can be adjusted and adapted into an appropriate means for testing a given substantive theory. In other words, the statistical methods are used to fit models that are tested against observed data patterns and are implemented such that issues of effect size, parsimony, and theoretical integration are emphasised (cf. null hypothesis testing).

Numerous recent techniques would fit within this modelling framework. For example, dyadic, triadic, and social relations paradigms are extremely useful to model the interactions among individuals (see e.g., Hawley \& Little, 1999; Kenny, 1990). These designs allow decomposition of the systematic sources of variance that contribute to individual behaviour; they also allow decomposition of the degree to which individuals contribute to the behaviour of the larger unit (i.e., dyed, triad, family, and so on). Similarly, multiple-group and multilevel designs are useful to compare and contrast the influences of different contexts as well as to partition the degree to which variability in behaviour is associated with contextual features versus individual differences (Little, 1997; Little et al., 2000). These techniques will be particularly useful to examine differences among groups and subgroups (i.e., typologies) of individuals.

In a related vein, recent advances in data imputation techniques and incomplete design estimation have tremendous potential for reshaping how we organise our data collection protocols. Various forms of intentionally missing designs can be conceived that would allow researchers to collect data in a more efficient and less costly manner and still be able to draw valid generalisations based on the whole sample of individuals and variables. For example, one might soon see a study of some large number of individuals but where any one individual responds to only a subset of the items of interest. With proper care, the missing subset of items could be veridically imputed (or the missing relationships estimated) such that conclusions and generalisations are drawn on the full set of individuals and variables (Graham \& Hofer, 2000; McArdle, 1994). Clearly, at this stage, the boundaries and limitations of such designs still need careful testing, but the potential of such intentionally missing designs is certainly an appealing possibility for large-scale contextualorganismic research programmes.

Finally, recent advances in general structural equation modelling procedures are beginning to overcome one of the more severe limitations of this class of modelling technique; namely, the presumption of linear relationships among continuous variables. Now, the procedures offer researchers the ability to specify latent interactions and nonlinear relationships as well as to model the relations among a mixture of different types of variables and constructs (see e.g., Little et al., 2000; Marcoulides \& Schumacker, 1996). Such advances will undoubtedly allow much greater precision in the manner by which theoretical propositions are specified, estimated, and tested.

\section{Broader implications of these themes}

From my perspective, a determined focus on these themes can yield a better understanding of the complexities of the relationships between context and self and how they influence successful development along the life course. These themes also have important implications for how programmes of research would be conceptualised and executed. For example, in order for these three themes to succeed in their potential to understand development, research programmes will need to be highly collaborative and designs will need to be both more encompassing of the venous microdivisions of the field; that is, more multidisciplinary in nature.

Regarding collaborative work, a major goal of the next era should be to pool the intellectual and talent capital of numerous researchers in order to ensure that the large-scale research programmes are conducted well. For example, a researcher on control and motivation can join forces with a specialist in personality and a specialist on emotion regulation to design a study that does more than just explore the interrelations among the separate categories of study, but rather, generates and tests meaningful hypotheses regarding the interface among the areas. Such approaches would advance our science by allowing us to contrast, reconcile, and integrate the plethora of microtheories that abound in developmental psychology.

Cross-cultural approaches are just one manifestation of collaborative work. Another type are the interdisciplinary network studies that integrate various disciplines in the social and behavioural sciences and cross-over into the biological and neurobiological realms (e.g., the Berlin Aging Study; Baltes \& Mayer, 1999). The model of the network will certainly be one instantiation of the successful research programme in the next century. Such a model will also impact the culture of psychological inquiry. For example, it is likely that fewer studies will emerge with single-authored credits. Instead, the results of research teams and networks will continue to propagate our journals (e.g., Grob, Little, Wanner, Wearing, \& Euronet, 1996).

Clearly, one development of the latter part of this century that will dramatically change how our research programmes are conducted is the advent of the Internet. Not only are we in close contact with colleagues from around the world, but also, the exchange of data, ideas, manuscripts, and so on is becoming increasingly seamless. Large-scale networks will be able to easily establish the connections among scholars that allow research programmes to aggressively model the agentic self in context. In addition, use of the Internet as a data collection medium will also allow larger and more broad-based studies to be efficiently conducted. Given the dramatic increases in the availability of computers and the Internet, the selection biases associated with Internet-based research will begin to dissipate once availability is more commonplace (or when access can be controlled by researchers, such as using random selection of participants in a networked school).

The type of research that I have outlined here lies at the cusp between basic and applied paradigms. Therefore, the results of studies that focus on these themes have tremendous potential to impact the field. From a basic research perspective, they allow strong tests of competing theoretical models and provide important integrative implications. From an applied perspective, the mass of knowledge that potentially can be gained would allow policy-oriented programmes the needed 
basis for designing interventions that are maximally effective. Such interventions would thereby reflect the complexities and multiple pathways in the development of the agentic self.

Alarger concern is clearly the degree to which we can make statements about causality in the context of quasi-experimental designs. As with any scientific method, threats to validity are numerous and, therefore, great caution is required. On the other hand, solid designs, good theory, and appropriate use of the analytic machinery that is available, has the potential to advance our knowledge base at an incredible rate. Moreover, multiple levels of analysis techniques and the judicious use of experimentation procedures can alleviate many of the concerns. However, in order to fully reap the benefits of contextual-organismic studies, I believe that training programmes will need to focus greater emphasis on both the basics and advances in quasi-experimental analytic techniques and expand instruction on correspondingly sophisticated designs.

In sum, behind every challenge is an opportunity. As a milestone for both societal and scientific development, the new millennium highlights the growing challenge of change and diversity. In my view, modelling the development of the agentic self in context is the opportunity that the millennium provides.

Manuscript received March 1999 Revised manuscript received September 1999

\section{References}

Baltes, P.B., \& Mayer, K.U. (Eds.) (1999). The Berlin aging study: Aging from 70 to 100. New York: Cambridge University Press.

Bronfenbrenner, U. (1995). Developmental ecology through space and time: A future perspective. In P. Moen, \& G.H. Elder, Jr. (Eds.), Examining lives in context: Perspectives on the ecology of human development (pp. 619-647). Washington, DC: American Psychological Association.

Gottlieb, G. (1997). Commentary: A systems view of psychobiological development. In D. Magnusson (Eds.), The lifespan development of individuals: Behavioral, neurobioloical, and psychosocial perspectives: A synthesis (pp. 76103). New York, NY: Cambridge University Press.

Graham, J.W., \& Hofer, S.M. (2000). Multiple imputation in multivariate research. In T.D. Little, K.U. Schnabel, \& J. Baumert (Eds.), Modeling longitudinal and multilevel data: Practical issues, applied approaches, and specific examples (pp. 201-218). Mahwah, NJ: Erlbaum.

Grob, A, Little, T.D., Wanner, B., Wearing, AJ., \& Euronet (1996). Adolescents' well-being and perceived control across fourteen sociocultural contexts. Journal of Personality and Social Psychology, 71, 785-795.

Hawley, P.H. (1999). The ontogenesis of social dominance: A strategy-based evolutionary perspective. Developmental Review, 19, 91-132.

Hawley, P.H., \& Little, T.D. (1999). On winning some and losing some: Social dominance in toddlers. Merrill Palmer Quarterly, 45, 185-214.

Hawley, P.H., Pasupahti, M., \& Little, T.D. (1999). Winning friends and influencing peers. Unpublished manuscript, Yale University.

Kenny, D.A (1990). Design issues in dyadic research. In C. Hendrick \& M.S. Clark (Eds.), Review of personality and social psychology: Research methods in personality and social psychology (Vol. 11, pp. 164-184). Newbury Park, CA: Sage.
Lerner, R.M. (1995). Developing individuals within changing contexts: Implications of developmental contextualism for human development research, policy, and programs. In T.A. Kindermann \& J. Valsiner (Eds.), Development of person-context relations (pp. 13-37). Hillsdale, NJ: Erlbaum.

Lerner, RM. (1996). Relative plasticity, integration, temporality, and diversity in human development: Adevelopmental contextual perspective about theory, process, and method. Developmental Psychology, 32, 781-786.

Lewis, M.D. (1997). Personality self-organization: Cascading constraints on cognition-emotion interaction. In A Fogel, M.C.D.P. Lyra, \& J. Valsiner (Eds.), Dynamics and indeterminism in developmental and social processes (pp. 193-216). Mahwah, NJ: Erlbaum.

Little, T.D. (1997). Mean and covariance structures (MACS) analyses of crosscultural data: Practical and theoretical issues. Multivariate Behavioral Research, $32,53-76$.

Little, T.D. (1998). Sociocultural influences on the development of children's action-control beliefs. In J. Heckhausen \& C.S. Dweck (Eds.), Motivation and self-regulation across the life span (pp. 281-315). New York: Cambridge University Press.

Little, T.D., Hawley, P.H., Henrich, C.C., \& Marsland, K. (in press). Three views of the agentic self: A developmental synthesis. In RM. Ryan \& E.L. Deci (Eds.), Handbook of self-determination research. Rochester, NY: University of Rochester Press.

Little, T.D., Schnabel, K.U., \& Baumert, J. (Eds.) (2000). Modeling longitudinal and multilevel data: Practical issues, applied approaches, and specific examples. Mahwah, NJ: Erlbaum.

Magnusson, D., \& Cairns, R.B. (1996) Developmental science: Toward a unified framework. In R.B. Cairns, G.H. Elder, Jr., \& J.E. Costello (Eds.), Developmental science (pp. 7-30). New York, NY: Cambridge University Press.

Marcoulides, G.A. \& Schumacker, R.E. (Eds.) (1996). Advanced structural equation modeling: Issues and techniques. Mahwah, NJ: Erlbaum.

McArdle, J.J. (1994). Structural factor analysis experiments with incomplete data. Multivariate Behavioral Research, 29, 409-454.

Mischel, W., \& Shoda, Y. (1995). A cognitive-affective system theory of personality: Reconceptualizing situations, dispositions, dynamics, and invariance in personality structure. Psychological Review, 102, 246-268.

Ryan, R.M. (1993). Agency and organization: Intrinsic motivation, autonomy, and the self in psychological development. In J.E. Jacobs (Ed.), Nebraska symposium on motivation, 1992: Developmental perspectives on motivation (Vol. 40, pp. 1-56). Lincoln, NE: University of Nebraska Press.

Ryan, R.M., Sheldon, K.M., Kasser, T., \& Deci, E.L. (1996). All goals are not created equal: An organismic perspective on the nature of goals and their regulation. In P.M. Gollwitzer \& J.A Bargh (Eds.), The psychology of action: Linking cognition and motivation to behavior (pp. 7-26). New York: Guilford Press.

Shanahan, M., Valsiner, J., \& Gottlieb, G. (1997). Developmental concepts across disciplines. In J. Tudge, M. Shanahan, \& J. Valsiner (Eds.), Comparisons in human development: Understanding time and context (pp. 1371). New York: Cambridge University Press.

Skinner, E.A (1995). Perceived control, motivation and coping. Thousand Oaks, CA: Sage.

Smith, J.S., \& Baltes, P.B. (1999). Trends and profiles of psychological functioning in very old age. In P.B. Baltes \& K.U. Maier (Eds.), The Berlin aging study: Aging from 70 to 100 (pp. 197-226). New York: Cambridge University Press.

Weisz, J.R. (1990). Development of control-related beliefs, goals, and styles in childhood and adolescence: A clinical perspective. In J. Rodin, C. Schooler \& W. Schaie (Eds.), Self-directedness: Cause and effects throughout the life course (pp. 19-49). Hillsdale, NJ: Erlbaum.

Widaman, K.F. (2000). Testing cross-group and cross-time constraints on parameters using the general linear model. In T.D. Little, K.U. Schnabel, \& J. Baumert (Eds.), Modeling longitudinal and multilevel data: Practical issues, applied approaches, and specific examples (pp. 163-186). Mahwah, NJ: Erlbaum. 